\title{
Reliable In-Plane Velocity Measurements With Magnetic Resonance Velocity Imaging
}

Haosen Zhang

Cleveland State University

Sandra S. Halliburton

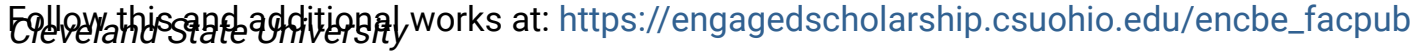

Part of the Biomedical Devices and Instrumentation Commons

Andan K. Venkatachari

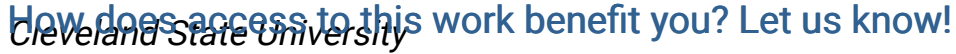

Publisher's Statement

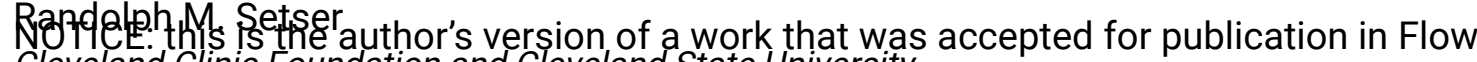
Cleveland Clinic Foundation and Cleveland State University

Measurement and Instrumentation. Changes resulting from the publishing process, such as

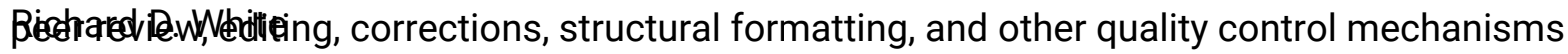
frlayehondbelipiqfegted atiqhis document. Changes may have been made to this work since it was submitted for publication. A definitive version was subsequently published in Flow

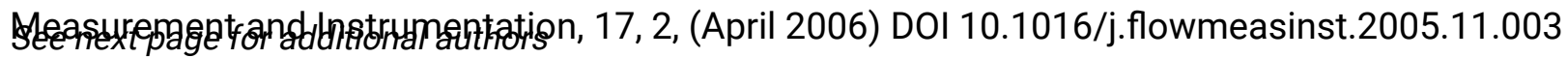

\section{Original Citation}

Zhang, H., Halliburton, S. S., Venkatachari, A. K., Setser, R. M., White, R. D., , \& Chatzimavroudis, G. P. (2006). Reliable in-plane velocity measurements with magnetic resonance velocity imaging. Flow Measurement and Instrumentation, 17(2), 75-80. doi:10.1016/j.flowmeasinst.2005.11.003

\section{Repository Citation}

Zhang, Haosen; Halliburton, Sandra S.; Venkatachari, Andan K.; Setser, Randolph M.; White, Richard D.; and Chatzimavroudis, George P., "Reliable In-Plane Velocity Measurements With Magnetic Resonance Velocity Imaging" (2006). Chemical \& Biomedical Engineering Faculty Publications. 74.

https://engagedscholarship.csuohio.edu/encbe_facpub/74

This Article is brought to you for free and open access by the Chemical \& Biomedical Engineering Department at EngagedScholarship@CSU. It has been accepted for inclusion in Chemical \& Biomedical Engineering Faculty Publications by an authorized administrator of EngagedScholarship@CSU. For more information, please contact library.es@csuohio.edu. 


\section{Authors}

Haosen Zhang, Sandra S. Halliburton, Andan K. Venkatachari, Randolph M. Setser, Richard D. White, and George P. Chatzimavroudis 


\title{
Reliable in-plane velocity measurements with magnetic resonance velocity imaging
}

\author{
Haosen Zhang ${ }^{\mathrm{a}, 1}$, Sandra S. Halliburton ${ }^{\mathrm{a}, \mathrm{b}}$, Anand K. Venkatachari ${ }^{\mathrm{a}}$, Randolph M. Setser ${ }^{\mathrm{a}, \mathrm{b}}$, \\ Richard D. White ${ }^{\mathrm{b}}$, George P. Chatzimavroudis ${ }^{\mathrm{a}, \mathrm{b}, *}$ \\ ${ }^{a}$ Laboratory of Biofluid Mechanics and Cardiovascular Imaging, Department of Chemical and Biomedical Engineering, Cleveland State University, Cleveland, \\ $\mathrm{OH} 44115$, United States \\ ${ }^{\mathrm{b}}$ Section of Cardiovascular Imaging, Division of Radiology, The Cleveland Clinic Foundation, Cleveland, OH 44115, United States
}

\section{Introduction}

Hydrogen-based magnetic resonance (MR) imaging is a standard imaging modality, with significant contribution to the clinical diagnosis of a variety of diseases. Its wide medical application is due to the significant amount of water in the human body. Inside the strong magnetic field of an MR scanner, the hydrogen nuclei precess at a frequency which is proportional to the strength of the magnetic field. If a linear magnetic field gradient is applied along any spatial direction, a corresponding linear change in the precession frequency of the nuclei will be induced. In fact, by applying such magnetic field gradients along all three spatial directions with proper timing, the position of the nuclei can be encoded into the characteristics (frequency-phase) of the acquired signal [1, 2]. An imaging sequence consists of the following steps: (a) slice selection and nuclei excitation; (b) phase-encoding; (c) frequency-encoding and signal acquisition. Each of these steps involves the application of one spatial magnetic field gradient; at the end, all three spatial gradients have been applied, causing the encoding of the spatial position of the nuclei into the signal. This acquired signal is digitized and the data are used to fill the frequency domain, often referred to as k-space. Then, an inverse 2-D Fourier transform reconstructs the image in the space domain. 
In addition to its well-documented ability as a reliable imaging modality, MR imaging provides quantitative information about flow velocity in medical and non-medical applications [3-9]. The major advantage of MR velocity imaging in medicine is its unique clinical ability to measure all three spatial directions of the velocity in an imaging slice. Thus, it provides information about the through-plane velocity as well as the in-plane velocity components in every pixel of the image. This is extremely helpful in order to study complicated velocity fields.

The ability of MR to measure flow velocity is based on the fact that the velocity of moving hydrogen nuclei can be encoded into the phase of the detected signal by applying bipolar magnetic field gradients [10]. By using the proper gradients, the resulted phase is proportional to the nuclei velocity. Each acquisition leads to two images, one based on the magnitude of the signal and one based on the phase of the signal. The latter is in fact the velocity image, because of the linear relationship between signal phase and nuclei velocity.

MR velocity imaging can accurately measure the throughplane velocity and quantify flow under both steady and pulsatile flow conditions, with errors typically within $\pm 5 \%$ [11-15]. Recently, we evaluated experimentally and clinically the potential of ultra-fast, segmented k-space MR velocity imaging for through-plane velocity measurements in tubes [16] and in the human aorta [17]. Our experimental results showed high accuracy (errors of less than $5 \%$ in the flow rate calculation) using segmented $\mathrm{k}$-space techniques with up to $9 \mathrm{k}$-space lines per segment. Our clinical results showed very close agreement between the segmented k-space ( 9 lines per segment) sequences and the conventional non-segmented sequence (difference of less than 5\%). Based on the confidence we obtained from these studies, the aim of this work was to investigate the reliability of MR velocity imaging in measuring the in-plane velocity under a variety of flow rates. Acquisition of reliable in-plane velocity data is very important considering the need to characterize and quantify two- and three-directional flow fields in medical and non-medical applications.

\section{Methods}

\subsection{Instrumentation, models, and flow set-up}

Steady water flow experiments were conducted in a $1.5 \mathrm{~T}$ Siemens Sonata whole-body MR scanner (Siemens Medical Solutions, Erlangen, Germany) with a maximum gradient strength of $40 \mathrm{mT} / \mathrm{m}$. MR velocity measurements were performed in three straight rigid PVC tubes with inner diameters of $14.7 \mathrm{~mm}$ (tube \#1), $20.2 \mathrm{~mm}$ (tube \#2), and $26.2 \mathrm{~mm}$ (tube \#3). The tubes were placed in a water-filled acrylic container in order to tune the scanner. Without the addition of water, the scanner failed to detect enough protons within the field of view to properly adjust the transmit/receive frequency. Steady flow studies were performed with flow rates ranging between 1.0 and $7.0 \mathrm{~L} / \mathrm{min}$ (Table 1). The Reynolds number $(R e)$ ranged between 1450 and 10100 . The true flow rate was known via pre-calibrated rotameters.
Table 1

\begin{tabular}{|c|c|c|c|c|}
\hline $\begin{array}{l}\text { Tube } \\
\#\end{array}$ & $\begin{array}{l}\text { Tube } \\
\text { diameter } \\
(\mathrm{mm})\end{array}$ & $\begin{array}{l}\text { Flow rate } \\
(\mathrm{L} / \mathrm{min})\end{array}$ & $\begin{array}{l}\text { Cross sectional average } \\
\text { velocity }(\mathrm{cm} / \mathrm{s})\end{array}$ & $N_{R e}$ \\
\hline \multirow{3}{*}{1} & \multirow{3}{*}{14.7} & 1.0 & 9.8 & 1450 \\
\hline & & 4.0 & 39.3 & 5800 \\
\hline & & 7.0 & 68.7 & 10100 \\
\hline \multirow{3}{*}{2} & \multirow{3}{*}{20.2} & 1.5 & 7.8 & 1570 \\
\hline & & 2.0 & 10.4 & 2100 \\
\hline & & 5.0 & 26.0 & 5250 \\
\hline 3 & 26.2 & 7.0 & 21.6 & 5670 \\
\hline
\end{tabular}

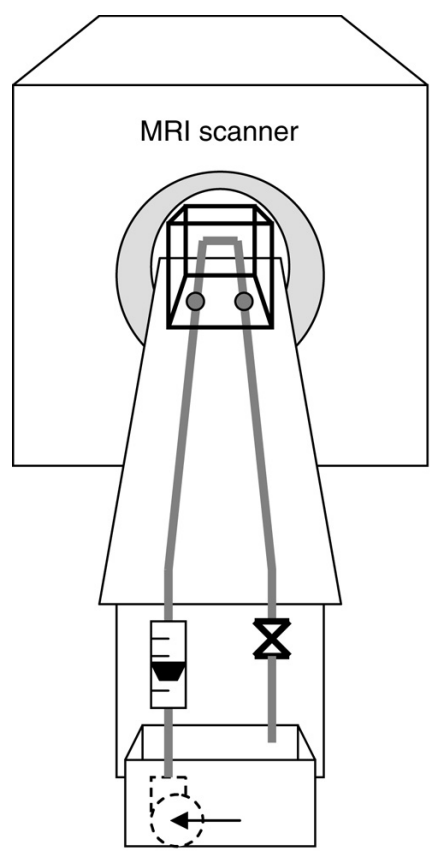

Fig. 1. The flow loop. The reservoir (bottom of figure) is placed far from the MR scanner, at the end of the patient table, to avoid any possible effects from the strong magnetic field on the pump and the rotameter. Fluid from the reservoir flows to the scanner using the submersible steady flow pump via PVC tubes. The test section is placed inside an acrylic container filled with water. The container is placed inside the MRI scanner with the location of interest at the center of the bore. The fluid follows the U-shaped tube and returns to the reservoir. The flow rate is controlled using a valve and is monitored using a precalibrated rotameter. No metallic objects should be placed close to the scanner to avoid accidents and interaction.

\subsection{Imaging procedure}

The test section (water-filled container with submerged straight tubes) was connected to the flow loop, and the entire system was inserted into the bore of the scanner with the testsection placed at the iso-center (Fig. 1). A phased-array receiver coil was used to cover the test section to improve image quality.

Initial scout images showed the exact location of the tubes in the scanner. Then, for each tube and flow rate, two MR velocity acquisitions were planned: one for an in-plane velocity measurement and one for a through-plane velocity measurement (to be used as a reference for comparison). For the through-plane velocity measurement, an imaging slice was placed at the iso-center of the scanner, perpendicular to the 
(a)

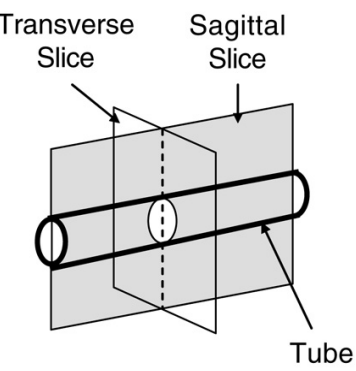

(b)

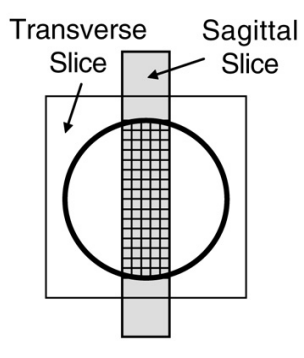

Fig. 2. (a) Transverse and sagittal slice orientations for through-plane and in-plane velocity acquisitions, respectively. (b) Since the pixel size in the transverse image was $1 \mathrm{~mm}$, five columns of pixels were selected to match the $5 \mathrm{~mm}$ sagittal slice thickness.

long axis of the tube under study in the transverse orientation. For the in-plane velocity measurement, an imaging slice was placed at the iso-center of the scanner, parallel to the long axis of the tube in the vertical (sagittal) orientation. The two slice orientations are shown in Fig. 2(a). The slices intersected along the vertical centerline of the tube (in fact, the intersection region was $5 \mathrm{~mm}$ wide encompassing the vertical tube centerline). Therefore, comparisons between the vertical centerline velocity profiles from these two acquisitions were possible.

Each through-plane and each in-plane velocity acquisition was performed three times using: (a) the conventional nonsegmented MR sequence with one $\mathrm{k}$-space line per segment (non-seg); (b) a segmented MR sequence with seven k-space lines per segment (seg-7); and (c) a segmented MR sequence with nine k-space lines per segment (seg-9). The difference between non-segmented and segmented k-space sequences is in the way k-space is filled. With the non-segmented procedure, only one line of $\mathrm{k}$-space is filled each time the imaging sequence is applied. For an image of $N \times N$ pixels, $N$ k-space lines are normally needed. Thus, to obtain the necessary number $N$ of $\mathrm{k}$-space lines for image reconstruction, data must be acquired over $N$ repetitions of the imaging sequence. This can be timeconsuming, and thus problematic, when the velocity has to be acquired at multiple locations, in all three spatial directions, or under unsteady flow conditions. With advancement in MR hardware and software, it has been possible to acquire multiple $\mathrm{k}$-space lines per imaging sequence. Instead of acquiring only one k-space line per sequence (non-segmented), a segment of $M$ k-space lines can be acquired per sequence. To fill all $N$ k-space lines, data must be acquired over $N / M$ sequences (instead of $N$ for the non-segmented sequence). The larger the number $(M)$ of lines per segment, the faster the acquisition. However, in unsteady flow cases, this leads to a decrease in the temporal resolution.

All of the velocity acquisitions were performed using a flip angle of $30^{\circ}$. The slice thickness was $5 \mathrm{~mm}$ and the field of view was $250 \times 250 \mathrm{~mm}^{2}$. A $75 \%$ rectangular field of view was used for the non-segmented acquisitions and a $56 \%$ rectangular field of view was used for the segmented acquisitions. The voxel size (after interpolation) was $1.0 \times 1.0 \times 5.0 \mathrm{~mm}^{3}$ in all cases. The velocity encoding value was $20-140 \mathrm{~cm} / \mathrm{s}$, depending on the magnitude of the flow. The echo time was varied between 3.1 and $5.6 \mathrm{~ms}$ (shortest possible based on

other imaging parameters). Under these imaging conditions and without gating, it takes $6 \mathrm{~s}$ for the non-segmented acquisitions and approximately $1 \mathrm{~s}$ for the segmented acquisitions. If the MR scanner is triggered and the data acquisition is gated in order to acquire several measurements throughout the flow cycle (necessary when the flow is pulsatile), it takes over $3 \mathrm{~min}$ for the non-segmented acquisitions and no more than $20 \mathrm{~s}$ for the non-segmented acquisitions. Considering the pulsatile nature of blood flow in the arteries, it is easy to appreciate the clinical importance of a reliable segmented k-space MR velocity imaging protocol.

\subsection{Image data analysis}

All (magnitude and phase) images were transferred to a work-station (Ultra-10, SUN Microsystems, Inc., Palo Alto, CA). The images were visualized using Transform (Version 3.4, Research Systems, Inc., Boulder, CO). A computer program converted the phase values of the phase images to velocity values based on the linearity between signal phase and proton velocity. The phase images were corrected for eddy currents [18] and for contributions due to Maxwell concomitant gradient terms [19]. The corrections were calculated from the known gradient waveforms. The lumen of the tubes was segmented manually in the transverse images. Then, the flow rate was calculated by summing up the products of "pixel velocity" and "pixel area". The accuracy of these flow rate calculations is related to the accuracy of the velocity profiles in the transverse images. To evaluate the in-plane velocity measurements, the sagittal in-plane velocity profiles were compared to the transverse through-plane velocity profiles in the intersection region of the transverse and sagittal images (which included the vertical tube centerline). Since the pixel size in the transverse image was $1 \mathrm{~mm}$, five columns of pixels (Fig. 2(b)) were selected (with the central column at the centerline) to match the $5 \mathrm{~mm}$ sagittal slice thickness. The velocities in these five columns were averaged to produce one column that covered the same portion of the tube as the sagittal slice.

Regression analysis, correlation analysis, and $t$-tests were performed to compare (a) the calculated and true flow rates; (b) the in-plane velocity with the through-plane velocity; and (c) the in-plane velocity profiles obtained using segmented and non-segmented techniques. Minitab (Version 13, Minitab, Inc., State College, PA) was used for the statistical analysis. A $p$-value $<0.05$ indicated significant difference.

\section{Results}

Fig. 3 shows magnitude and phase (velocity) images acquired with a transverse slice and a sagittal slice. The images were of sufficient quality to segment the lumen of the tube and perform quantitative analysis.

The through-plane measurements provided accurate flow rate results for all sequences as shown from the small errors in the calculated flow rates compared to the true flow rates (errors of $7.5 \pm 3.8 \%, 5.1 \pm 5.1 \%$, and $4.7 \pm 5.8 \%$ for the $n o n-s e g$, seg- 7 , 

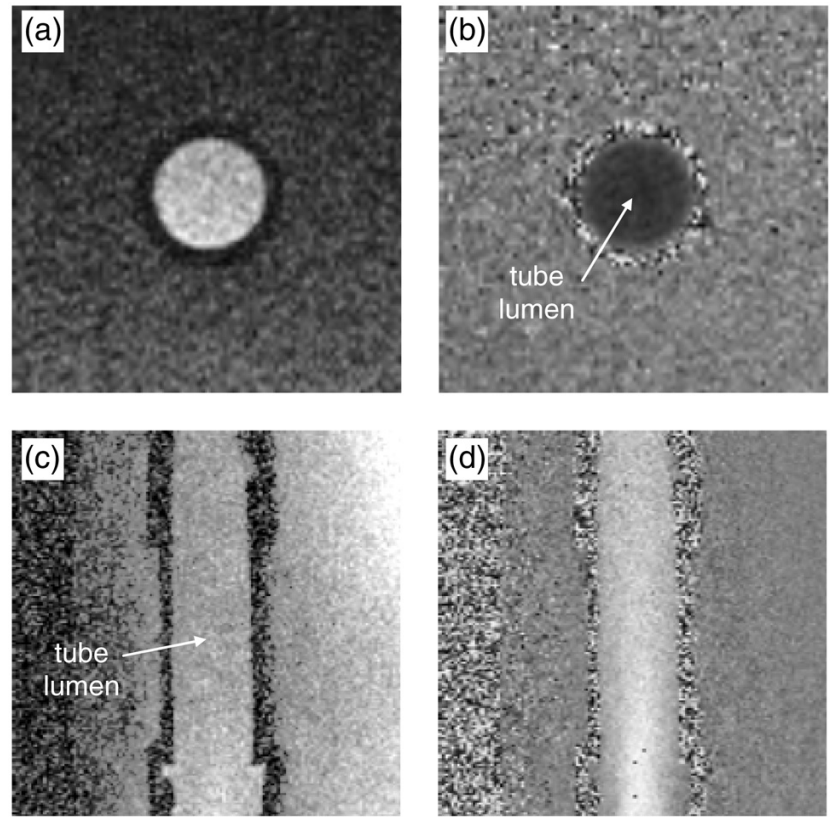

Fig. 3. (a) Magnitude transverse image; (b) phase transverse image; (c) magnitude sagittal image; and (d) phase sagittal image.

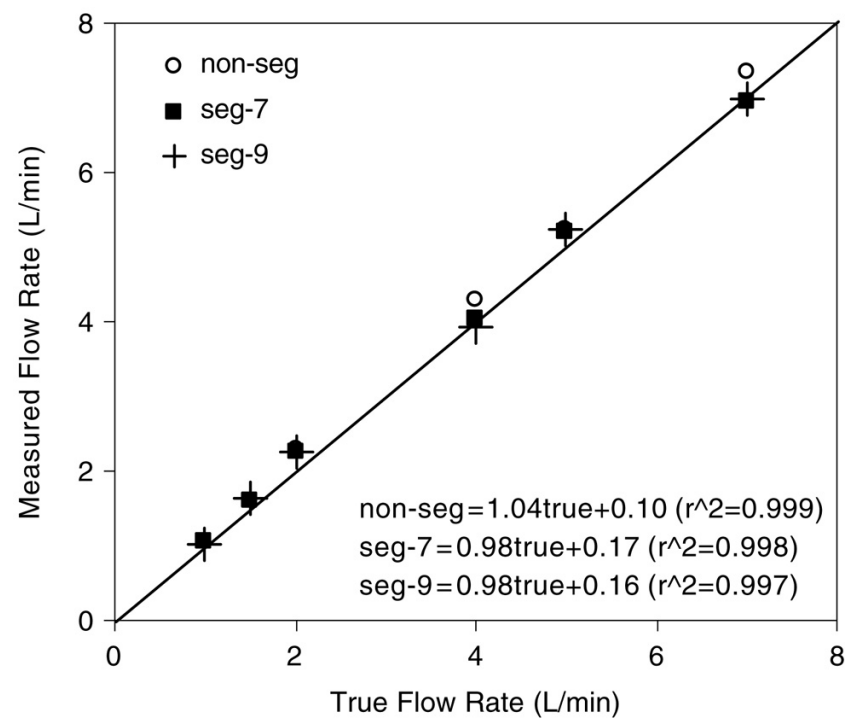

Fig. 4. Comparison of the measured flow rates with the true flow rates.

and seg-9 sequence, respectively), and from regression analysis (Fig. 4, $r^{2} \geq 0.99, p$-values $\left.=0.00\right)$. These errors were not statistically different from each other as shown through $t$-tests $(p>0.05)$. The centerline velocity profiles in a single column of pixels in the transverse images showed the well-known features of laminar and non-laminar flow. Laminar flow cases exhibited parabolic-like profiles, with a centerline velocity approximately twice (average of 1.97 times) as large as the cross-sectional average velocity. Non-laminar flow exhibited flatter profiles with the centerline velocity significantly less than twice (between 1.3 and 1.7 times) the average velocity.

Good agreement was found between the through-plane and the in-plane velocity profiles at various flow rates (Fig. 5). Fig. 5(a) corresponds to a true flow rate of $1.0 \mathrm{~L} / \mathrm{min}$
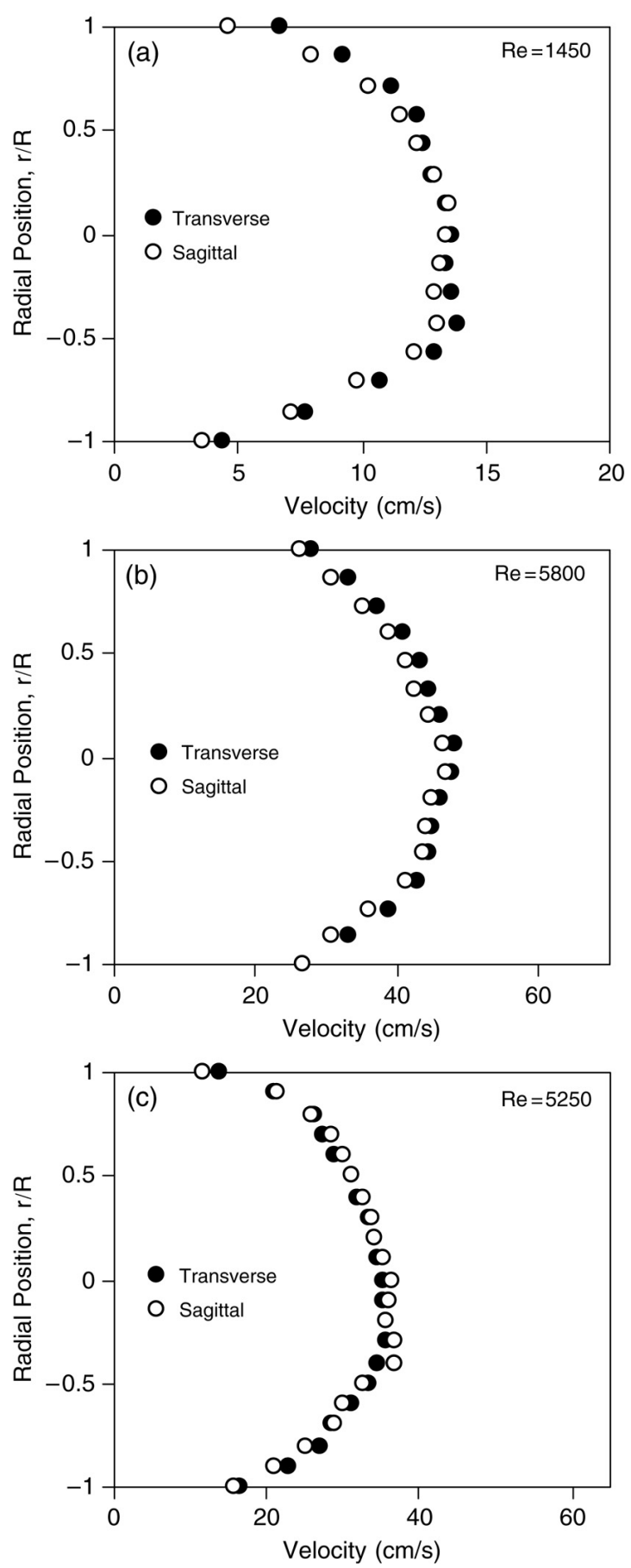

Fig. 5. Comparison of through-plane with in-plane velocity profiles at: (a) $1.0 \mathrm{~L} / \mathrm{min}$ (tube \#1, Re = 1450); (b) $4.0 \mathrm{~L} / \mathrm{min}$ (tube \#1, $R e=5800$ ); and (c) $5.0 \mathrm{~L} / \mathrm{min}$ (tube \#2, Re $=5250$ ).

$(\operatorname{Re}=1450)$. The profiles, both acquired with the seg-7 sequence, agree closely. The difference (defined as [sagittal transverse]/transverse $\times 100$ across the profiles except for pixels at $r / R= \pm 1$ ) is $-5.0 \pm 4.4 \%$ (mean \pm standard deviation (SD) across the profiles). Fig. 5(b) shows the through-plane and inplane velocity profiles for a true flow rate of $4.0 \mathrm{~L} / \mathrm{min}(R e=$ $5800)$. Both profiles were measured with the seg-9 sequence. The profiles agree closely, with a difference of $-4.2 \pm 2.0 \%$ (mean \pm SD across the profiles). A similar agreement can be 

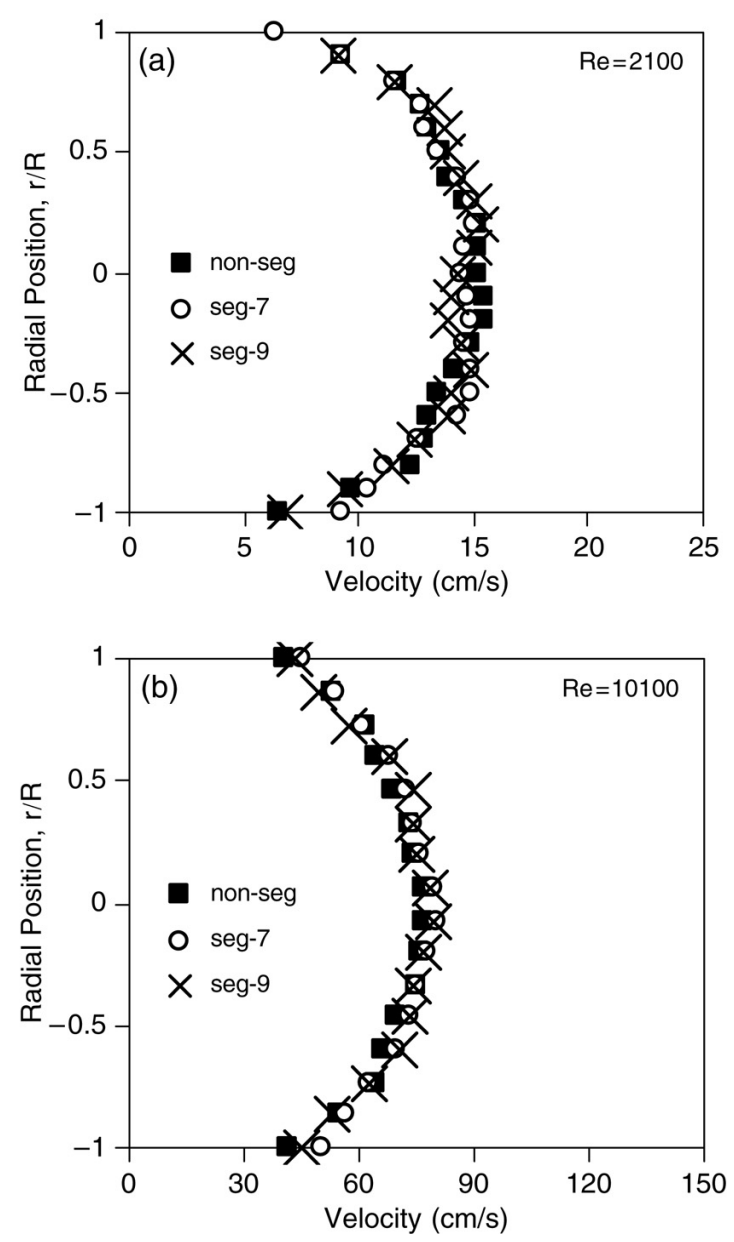

Fig. 6. Comparison between in-plane velocity profiles for all sequences used at: (a) $2.0 \mathrm{~L} / \mathrm{min}$ (tube \#2, $R e=2100$ ) and (b) $7.0 \mathrm{~L} / \mathrm{min}$ (tube $\# 1, R e=10100$ ).

seen in Fig. 5(c) that shows the through-plane and in-plane velocity profiles for a flow rate of $5.0 \mathrm{~L} / \mathrm{min}(R e=5250)$ using the non-seg sequence. The average difference between the profiles is $0.3 \pm 3.6 \%$ (mean $\pm \mathrm{SD}$ across the profiles). Strong correlation between the through-plane and in-plane profiles was found in all cases (Pearson correlation coefficients of $0.99,1.00$, and 0.99 for the profiles in Fig. 5(a)-(c), respectively). Similar levels of agreement were found for the rest of the flow rates tested (absolute average difference of $6 \%$ between throughplane and in-plane profiles).

Comparison between the velocity profiles from the segmented and the non-segmented sequences showed close agreement. Fig. 6 shows the in-plane profiles from all three sequences, for a flow rate of $2.0 \mathrm{~L} / \mathrm{min}(R e=2100)$ and $7.0 \mathrm{~L} / \mathrm{min}(R e=10100)$. For the lower flow rate, the differences across the profiles were as follows: seg-7 vs. nonseg $=0.1 \pm 5.2 \%$; seg-9 vs. non-seg $=-0.5 \pm 5.2 \%$; seg- 9 vs. seg-7 $=-0.5 \pm 4.0 \%$. The Pearson correlation coefficients were 0.96 between the non-seg and the seg-7 profiles, 0.97 between the non-seg and the seg- 9 profiles, and 0.97 between the seg- 9 and the seg-7 profiles. For the higher flow rate, the differences were: $\operatorname{seg}-7$ vs. non-seg $=2.5 \pm 2.5 \%$; seg-9 vs. non-seg $=1.0 \pm 4.3 \%$; seg- 9 vs. seg-7 $=-1.5 \pm 2.8 \%$. The Pearson correlation coefficients were 0.98 between the non-seg

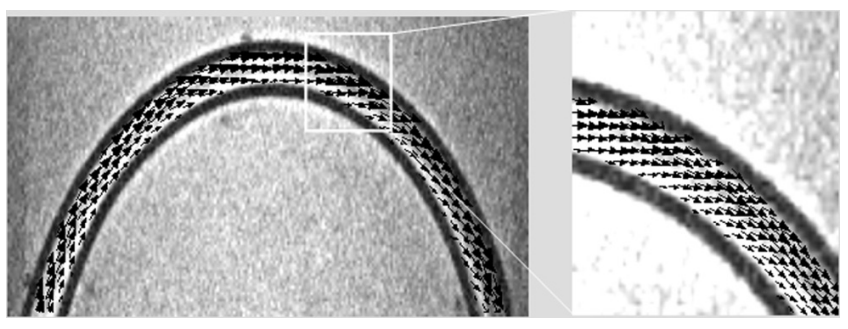

Fig. 7. Left: a velocity vector plot constructed by measuring and combining the two in-plane velocity components of water as it flows through a curved tube; Right: magnification immediately downstream of the top of the arch.

and the seg-7 profiles, 0.98 between the non-seg and the seg-9 profiles, and 0.99 between the seg- 9 and the seg-7 profiles.

\section{Discussion}

Acquisition of the in-plane velocity components can provide useful information about two- and three-directional flow fields. Fig. 7 shows an example of flow in a curved tube. This vector plot is a result of combining the two in-plane velocity measurements (superior-inferior and left-right directions). Although such velocity fields can also be obtained using laser Doppler anemometry or digital particle imaging velocimetry, MR is the only technique to assess two- and three-directional flow patterns in the human body, because it is currently the only clinical technique to provide all three spatial velocity components in an imaging slice. This unique clinical feature of MR has led to more sophisticated methodologies that include multi-slice schemes such as the recently-developed control volume method for the quantification of flows through orifices [20,21]. In such cases, having a rapid technique for velocity acquisitions is necessary. Otherwise, the long duration of scanning makes these applications impractical.

Despite its established accuracy for through-plane velocity measurements, there have been only a few experimental studies evaluating MR velocity imaging for in-plane velocity measurements [22-24]. Because of the difficulty to apply another technique (such as laser Doppler anemometry, digital particle image velocimetry, or Doppler ultrasound) in the MR scanner, it is difficult to compare the MR in-plane velocity data with velocity data acquired during the same experiment with another experimental technique. The reliability of throughplane MR velocity measurements has been extensively shown to be very high. This was confirmed in this study as well, by examining the through-plane velocity profiles in a single column along the tube centerline in the transverse images, showing the well-known features of laminar (parabolic-like profiles, with a centerline velocity approximately twice as large as the cross-sectional average velocity) and non-laminar flow (flatter profiles with the centerline velocity significantly less than twice the average velocity). Therefore, in this study we compared in-plane MR data with through-plane MR data (considered as the reference data), during the same experiment and at the same location, to obtain useful information about the reliability of MR for in-plane velocity measurements.

It should be noted that the through-plane and in-plane velocity profiles compared in this study are not the true 
vertical tube centerline profiles. Although the region where the transverse and sagittal slices meet encompasses the vertical tube centerline, this region is $5 \mathrm{~mm}$ wide (Fig. 2). As previously reported [22,24], the thickness of the slice affects the measured in-plane velocity values. If it is of interest to measure the centerline velocity, use of a very thick slice may cause errors in the velocity as high as $33 \%$ [24]. This should not affect the importance of the findings of this study, since the aim was not to measure the centerline velocity but to compare the in-plane and through-plane velocity profiles in the same region. This is the reason for which there was a match between the sagittal slice thickness $(5 \mathrm{~mm})$ and the number of columns (five) of pixels in the transverse image. Despite any imperfections in the slice profiles, the agreement found between the velocity profiles provides useful information about the reliability of segmented $\mathrm{k}$-space MR in measuring the in-plane velocity.

This study evaluated MR velocity imaging in measuring in-plane velocity under steady flow conditions. Despite the fact that the majority of non-medical flow applications involve steady flow conditions, our next step will be to extend the investigation to unsteady flows and consider additional factors, such as the connection between $\mathrm{k}$-space segmentation and temporal resolution.

\section{Conclusion}

Steady flow experiments in straight rigid tubes showed that magnetic resonance velocity imaging can provide inplane velocity measurements that agree closely with throughplane velocity measurements at the same location. k-space segmentation provides ultra-fast acquisition of reliable in-plane velocity data, which is of importance considering the increasing need for faster, non-invasive, three-directional flow velocity measurements.

\section{Acknowledgments}

Support by Cleveland State University and by Siemens Medical Solutions is greatly appreciated.

\section{References}

[1] Haacke EM, Brown RW, Thompson MR, Venkatesan R. Magnetic resonance imaging: physical principles and sequence design. New York: Wiley; 1999.

[2] Bushberg JT, Seibert JA, Leidholdt Jr EM, Boone JM. The essential physics of medical imaging. 2nd ed. Philadelphia: Lippincott Williams \& Wilkins; 2001

[3] Mansfield P, Bowtell R, Blackband S, Guilfoyle DN. Magnetic resonance imaging: Applications of novel methods in studies of porous media. Magn Reson Imag 1992;10:741-6.

[4] Pelc LR, Pelc NJ, Rayhill SC, Castro LJ, Glover GH, Herfkens RJ et al. Arterial and venous blood flow: Noninvasive quantitation with MR imaging. Radiology 1992;185:809-12.

[5] Bogren HG, Buonocore MH. Blood flow measurements in the aorta and major arteries with MR velocity mapping. J Magn Reson Imag 1994;4: $119-30$.

[6] Corbett AM, Phillips RJ, Kauten RJ, McCarthy KL. Magnetic resonance imaging of concentration and velocity profiles of pure fluids and solid suspensions in rotating geometries. J Rheol 1995;39:907-24.

[7] Chatzimavroudis GP, Walker PG, Oshinski JN, Franch RH, Pettigrew RI, Yoganathan AP. Slice location dependence of aortic regurgitation measurements with MR phase velocity mapping. Magn Reson Med 1997; 37:545-51.

[8] Britton MM, Callaghan PT. NMR velocimetry study of the temperature dependent rheology of butter, semisoft butter and margarine. J Text Stud 2000;31:245-55.

[9] Mantle MD, Sederman AJ, Gladden LF. Single- and two-phase flow in fixed-bed reactors: MRI flow visualisation and lattice-Boltzmann simulations. Chem Eng Sci 2001;56:523-9.

[10] Moran PR. A flow velocity zeugmatographic interlace for NMR imaging in humans. Magn Reson Imag 1982;1:197-203.

[11] Bryant DJ, Payne JA, Firmin DN, Longmore DB. Measurement of flow with NMR imaging using a gradient pulse and phase difference technique. J Comp Assist Tomogr 1984;8:588-93.

[12] Meier D, Maier S, Bosiger P. Quantitative flow measurements on phantoms and on blood vessels with MR. Magn Reson Med 1988;8: 25-34.

[13] Frayne R, Steinman DA, Ethier CR, Rutt BK. Accuracy of MR phase contrast velocity measurements for unsteady flow. J Magn Reson Imag 1995;5:428-31.

[14] Polzin JA, Frayne R, Grist TM, Mistretta CA. Frequency response of multi-phase segmented k-space phase-contrast. Magn Reson Med 1996; 35:755-62.

[15] Moser KW, Kutter EC, Georgiadis JG, Buckius RO, Morris HD, Torczynski JR. Velocity measurements of flow through a step stenosis using magnetic resonance imaging. Exp Fluids 2000;29:438-47.

[16] Zhang H, Halliburton SS, Moore JR, Simonetti OP, Schvartzman PR, White RD et al. Accurate quantification of steady and pulsatile flow with segmented k-space magnetic resonance velocimetry. Exp Fluids 2002;33: 458-63.

[17] Chatzimavroudis GP, Zhang H, Halliburton SS, Moore JR, Simonetti OP, Schvartzman PR et al. Clinical blood flow quantification with segmented k-space magnetic resonance phase velocity mapping. J Magn Reson Imag 2003;17:65-71.

[18] Walker PG, Cranney GB, Scheidegger MB, Waseleski G, Pohost GM, Yoganathan AP. Semiautomated method for noise reduction and background phase error correction in MR phase velocity data. J Magn Reson Imag 1993;3:521-30.

[19] Bernstein MA, Zhou XHJ, Polzin JA, King KF, Ganin A, Pelc NJ et al. Concomitant gradient terms in phase contrast MR: Analysis and correction. Magn Reson Med 1998;39:300-8.

[20] Walker PG, Oyre S, Pedersen EM, Houlind K, Guenet FSA, Yoganathan AP. A new control volume method for calculating valvular regurgitation. Circulation 1995;92:579-86.

[21] Chatzimavroudis GP, Oshinski JN, Pettigrew RI, Walker PG, Franch RH, Yoganathan AP. Quantification of mitral regurgitation with magnetic resonance phase velocity mapping using a control volume method. J Magn Reson Imag 1998;8:577-82.

[22] Duerk JL, Pattany PM. Inplane flow velocity quantification along the phase encoding axis in MRI. Magn Reson Imag 1988;6:321-33.

[23] Siegel JM, Oshinski JN, Pettigrew RI, Ku DN. The accuracy of magnetic resonance phase velocity measurements in stenotic flow. J Biomech 1996; 29:1665-72.

[24] Kraft KA, Fei DY, Fatouros PP. Quantitative phase-velocity MR imaging of inplane laminar flow: Effect of fluid velocity, vessel diameter, and slice thickness. Med Phys 1992;19:79-85. 